\title{
Comunidade de mosquitos (Diptera, Culicidae) em recipientes antrópicos introduzidos em área rural e urbana da região metropolitana de Curitiba, Paraná, Brasil ${ }^{1}$
}

\author{
Daniéla C. Calado ${ }^{2,3}$ \\ Mário Antônio Navarro da Silva ${ }^{2}$
}

\begin{abstract}
Community of mosquitoes (Diptera, Culicidae) in artificial introduced containers in rural and urban area in region of Curitiba, Paraná, Brazil. Immature stages of Culicidae were collected in artificial containers in three conditions, between November 1997 and November 1998, in the rural area of São José dos Pinhais, Paraná, Brazil. Eight species were identified: Culex (Culex) eduardoi Casal \& Garcia, 1968, Culex (Culex) acharistus Root, 1927, Culex (Culex) group coronator, Culex (Lutzia) bigoti Bellardi, 1962, Aedes (Protomacleaya) terrens (Walker, 1856), Limatus durhamii Theobald, 1901, Txorhynchites theobaldi (Dyar \& Knab, 1906) and Phoniomyia sp. The Berger \& Parker index was obtained for each condition and kind of container. These results were compared with those of SiLVA \& Lozover (1996) in the urban area of Curitiba. A greater number of species was observed in the rural area, with different dominance under each condition. The introduction of artificial containers can not show the number of Culicidae species that are present in an area, but it can indicate the species that show the greatest adaptability to environmental modifications produced by human activity.

KEY WORDS. Culicidae, artificial containers, ecology
\end{abstract}

As áreas rurais, embora possam estar em contato com o ambiente silvestre, são fortemente influenciadas pelo rápido processo de urbanização, onde o crescimento populacional e habitacional, geram modificações ambientais capazes de contribuir para o estabelecimento de espécies de mosquitos melhor adaptadas ao ambiente antrópico. Tais alterações, podem determinar a redução de criadouros naturais e o aumento da produção de descartes, que passam a atuar como criadouros alternativos para muitas espécies de culicídeos. As mudanças ecológicas conseqüentes do desenvolvimento agrícola, talvez sejam o determinante que age com maior freqüência no aparecimento de vetores emergentes, por colocar o meio antrópico em contato mais estreito com as populações vetoras, as comunidades constituídas por elas e as dos agentes infecciosos (FORATTINI 1998).

A exploração de recipientes artificiais foi observada para várias espécies de culicídeos. Silva \& MENEzes (1996) relatam a presença de Aedes scapularis (Rondani, 1848) em lata abandonada próxima à uma mata residual no Município de Sertaneja, norte do Paraná, e destacam a possibilidade de novo comportamento

1) Contribuição número 1222 do Departamento de Zoologia, Universidade Federal do Paraná.

2) Departamento de Zoologia, Universidade Federal do Paraná. Caixa Postal 19020, 81531-990 Curitiba, Paraná, Brasil.

3) Bolsista CNPq. E-mail: dcalado@bio.ufpr.br 
desta espécie. ForatTini et al. (1998) encontraram formas imaturas de Anopheles (Kerteszia) bellator Dyar \& Knab, 1906 e Anopheles argyritarsis Robineau-Desvoidy, 1827 em criadouros experimentais instalados com a finalidade de verificar a evolução desses habitats no que concerne a presença de formas imaturas. LUZ et al. (1987) encontraram imaturos de Anopheles (Kerteszia) bambusicolus Komp, 1937 em pneu abandonado no interior do Parque Nacional do Iguaçu (Foz do Iguaçu, Estado do Paraná). Estes autores destacam, frente as transformações ambientais ocorridas na região, uma possível mudança de hábito desse anofelino, indicando maior valência ecológica da espécie.

A introdução de artefatos antrópicos em condições de armazenar água, em área rural ou ambientes de mata em região urbanizada, podem levar ao encontro de culicídeos em processo de mudança quanto aos tipos de criadouros preferencialmente utilizados (SIlva \& Lozover 1996). Podendo indicar plasticidade genética que os direcione evolutivamente no sentido da domiciliação LOPES (1997).

O objetivo deste trabalho foi estudar a ecologia de culicídeos que exploram recipientes artificiais introduzidos em área rural do Município de São José dos Pinhais e compará-la com os dados obtidos por SiLVA \& LOZOVEI (1996) em área urbana de Curitiba.

\section{MATERIAL E MÉTODOS}

As coletas em recipientes artificiais introduzidos foram realizadas em área

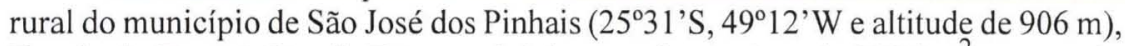
Estado do Paraná, Brasil. Este município possui uma área de $899 \mathrm{~km}^{2}$, ocupando $10,63 \%$ da região metropolitana de Curitiba (COMEC 1984). A área onde foram instalados os recipientes artificiais é representada por uma propriedade rural particular com 23 hectares de extensão, inserida na localidade da Colônia Marcelino.

As coletas de imaturos foram realizadas quinzenalmente, no período de novembro de 1997 a novembro de 1998, em recipientes artificiais de origem antrópica, constituídos de três tipos: a) pneu de automóvel, b) vasos de cerâmica e c) vasos de plástico. Os pneus utilizados, em posição vertical apresentavam capacidade para aproximadamente $3000 \mathrm{ml}$ de água. Os vasos de plásticos apresentavam coloração preta, com $18 \mathrm{~cm}$ de diâmetro superior, $18 \mathrm{~cm}$ de altura e capacidade para $3.300 \mathrm{ml}$ de água. Os vasos de cerâmica possuíam coloração marrom, com $23 \mathrm{~cm}$ de diâmetro superior, $20 \mathrm{~cm}$ de profundidade e capacidade máxima para $5.100 \mathrm{ml}$. No momento da instalação, os recipientes foram preenchidos até a capacidade máxima descrita acima.

Os recipientes foram instalados no interior da mata e próximos à habitação humana sob duas condições, sombreamento e expostos à insolação direta em área aberta. Em cada condição foram utilizados três tipos de recipientes. Os recipientes instalados no interior da mata ficavam distantes aproximadamente $20 \mathrm{~m}$ da borda da mata e aqueles sujeitos às condições de sombreamento e insolação direta no peridomicílio, ficavam à cinco metros da habitação. Em cada um dos habitats referidos, foi formado um conjunto de nove recipientes, com três repetições de cada tipo, ou seja, três pneus, três vasos de cerâmica e três vasos de plástico, colocados 
alternadamente para que recipientes de mesma natureza não ficassem próximos. Estes criadouros ficavam distantes três metros um do outro, constituindo três linhas e três colunas.

Nas amostragens dos imaturos, a água dos criadouros foi transferida para bandejas, de onde eram retiradas somente larvas de quarto instar e pupas. O restante do material foi devolvido ao criadouro de origem, completando o volume quando necessário, com água de poço artesiano.

Em laboratório, parte das larvas e todas as pupas eram transferidas para recipientes individuais, visando a obtenção dos adultos. A outra parte das larvas foi sacrificada e acondicionada em álcool a $80 \%$.

A classificação dos exemplares seguiu a proposta sistemática de HARBACH \& KiTCHING (1998) para Culicidae. Lâminas permanentes e exemplares adultos foram depositados na Coleção de Entomologia Pe. J.S. Moure, Departamento de Zoologia, Universidade Federal do Paraná (DZUP).

$\mathrm{Na}$ análise dos dados, foi utilizado o Índice de Dominância de Berger \& Parker, para cada tipo de recipiente instalado nos três ecótopos da área de estudo (MAGURRAN 1988). Para a comparação com a ocupação de recipientes artificiais de mesma constituição, em área urbana, foram utilizados os dados apresentados por Silva \& Lozovei (1996). Trabalho realizado comparando dois ecótopos, interior de capão de mata e área adjacente a esta mata, na área urbana de Curitiba.

Os dados meteorológicos foram obtidos em planilhas das observações meteorológicas da Estação número 83842 do Sistema Meteorológico do Paraná (Simepar), do Campus Universitário do Centro Politécnico da Universidade Federal do Paraná, localizada nas coordenadas $25^{\circ} 26^{\prime} \mathrm{S}, 49^{\circ} 16^{\prime} \mathrm{W}$ e $923,50 \mathrm{~m}$ de altitude, estação mais próxima da área de estudo. Para análise e cálculo das médias e somatórios das variáveis ambientais, os dados foram tomados entre coletas sucessivas.

\section{RESULTADOS E DISCUSSÃO}

Foram coletados 5500 imaturos, deste total, $64,29 \%$ foram obtidos no peridomicílio com sombreamento, $20,15 \%$ no interior de mata e $15,55 \%$ no peridomicílio sem sombreamento. Oito espécies de culicídeos foram identificadas: Culex (Culex) eduardoi Casal \& Garcia, 1968, Culex (Culex) acharistus Root, 1927, Culex (Culex) grupo coronator (coronator e/ou usquatus), Culex (Lutzia) bigoti Bellardi, 1862, Aedes (Protomacleaya) terrens (Walker, 1856), Limatus durhamii Theobald, 1901, Toxorhynchites (Lynchiella) theobaldi (Dyar \& Knab, 1906) e Phoniomyia sp. O gênero Culex foi encontrado em todos os tipos de criadouros utilizados, pneu, plástico e cerâmica, enquanto os demais foram coletados somente em pneu (Tab. I).

Durante o desenvolvimento da investigação as condições meteorológicas, registradas para o período entre as coletas sucessivas, foram as seguintes: as médias das temperaturas mínima, média e máxima foram respectivamente, $13,78(3,27)^{\circ} \mathrm{C}$, $17,76(3,31)^{\circ} \mathrm{C}$ e $22,07(3,62)^{\circ} \mathrm{C}$. Com a média da temperatura máxima mais elevada ocorrendo em fevereiro $29,56(1,20)^{\circ} \mathrm{C}$ e a média da mínima alcançando 8,01 $(1,68)^{\circ} \mathrm{C}$ no mês de junho. Em relação a dados diários a temperatura mais elevada foi registrada para o mês de dezembro $\left(32^{\circ} \mathrm{C}\right)$, e a mínima em junho $\left(2,1^{\circ} \mathrm{C}\right)$. A 
precipitação pluviométrica média, também para o período entre coletas sucessivas, atingiu 83,88 (67,00) $\mathrm{mm}$, sendo a acumulada para os 13 meses de $2179,52 \mathrm{~mm}$. O período entre as coletas foi marcado pelo predomínio de baixas temperaturas e níveis elevados de precipitação pluviométrica (Fig. 1).

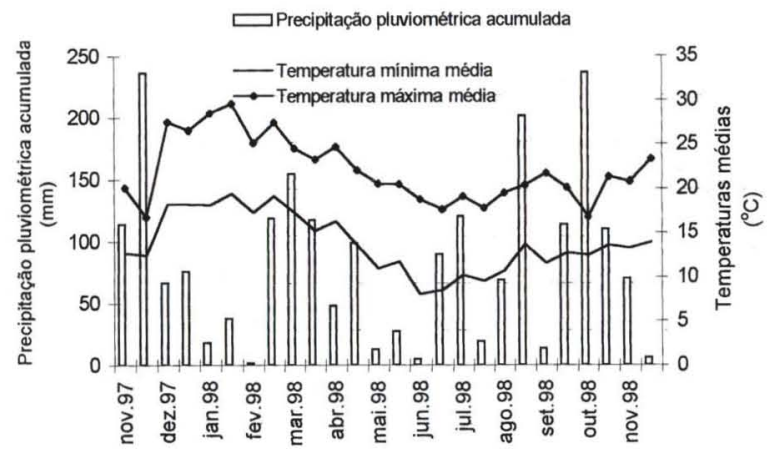

Fig. 1. Temperaturas máximas e minimas médias $\left({ }^{\circ} \mathrm{C}\right)$ e precipitação pluviométrica acumulada $(\mathrm{mm})$ entre coletas sucessivas da estação Centro Politécnico, Universidade Federal do Paraná, Curitiba, Paraná, de novembro de 1997 a novembro de 1998.

Em relação a natureza dos recipientes, o pneu abrigou a maior porcentagem de imaturos, $42,2 \%$. Os recipientes constituídos por plástico apresentaram 30,0\% de imaturos e os de cerâmica abrigaram a menor porcentagem $27,8 \%$. No pneu também ocorreu o maior número de espécies, seguido do plástico e cerâmica. Embora o número de espécies coletadas tenha sido superior para o plástico em relação a cerâmica, no primeiro foi coletada apenas uma espécie a mais, com número reduzido de exemplares (Tab. I).

Tabela I. Total de imaturos de culicídeos coletados nos três tipos de recipientes introduzidos, em três condições distintas, em área rural de São José dos Pinhais, Paraná, Brasil, de novembro de 1997 a novembro de 1998. (IMT) Interior de Mata, (CSL) peridomicílio sem sombreamento, (CSB) peridomicilio com sombreamento.

\begin{tabular}{|c|c|c|c|c|c|c|c|c|c|c|}
\hline \multirow{2}{*}{ Espécies } & \multicolumn{3}{|c|}{ Pneu } & \multicolumn{3}{|c|}{ Cerâmica } & \multicolumn{3}{|c|}{ Plástico } & \multirow{2}{*}{ Tota } \\
\hline & IMT & CSL & CSB & IMT & CSL & CSB & IMT & CSL & CSB & \\
\hline Aedes terrens & 29 & 0 & 42 & 0 & 0 & 0 & 0 & 0 & 0 & 71 \\
\hline Culex grupo coronator & 0 & 15 & 1 & 0 & 175 & 0 & 0 & 219 & 0 & 410 \\
\hline Culex bigoti & 0 & 1 & 20 & 0 & 0 & 0 & 0 & 0 & 7 & 28 \\
\hline Culex eduardoi & 495 & 299 & 957 & 116 & 33 & 945 & 297 & 0 & 1045 & 4187 \\
\hline Culex acharistus & 45 & 105 & 146 & 115 & 0 & 144 & 0 & 1 & 79 & 633 \\
\hline Limatus durhamii & 1 & 8 & 130 & 0 & 0 & 0 & 0 & 0 & 0 & 139 \\
\hline Phomiomyia sp. & 1 & 0 & 0 & 0 & 0 & 0 & 0 & 0 & 0 & 1 \\
\hline Toxorhynchites theobaldi & 9 & 0 & 20 & 0 & 0 & 0 & 0 & 0 & 0 & 29 \\
\hline Total & 580 & 428 & 1316 & 231 & 208 & 1089 & 297 & 220 & 1131 & \\
\hline Total de imaturos & & 2324 & & & 1528 & & & 1648 & & 5500 \\
\hline
\end{tabular}


No peridomicílio com sombreamento, foi observado o maior número de recipientes positivos para larvas de quarto instar e pupas, durante grande parte do ano, com exceção da primavera onde a média foi semelhante àquela obtida para o interior de mata. Do total de recipientes amostrados, no peridomicílio com sombreamento foram observados $114(16,2 \%)$ recipientes positivos, seguido pelo interior de mata com $66(9,4 \%)$ e pela área sem sombreamento no peridomicílio $61(8,7 \%)$. Durante o verão e inverno, a média de ocupação dos recipientes foi significativamente diferente entre os ecótopos, com predomínio no peridomicílio com sombreamento. Nesta área também foi observada a maior densidade, 31,01 imaturos por recipiente, podendo ser considerada como a área mais adequada ao desenvolvimento dos imaturos de culicídeos. No peridomicílio com sombreamento, a matéria orgânica presente nos criadouros era representada por grande quantidade de folhas que forneciam fonte de nutrientes às larvas. BEIER et. al. (1983) destacam que pneus mantidos em área sombreada recebem folhas e são enriquecidos com água que penetra através da copa das árvores, resultando em maior número de imaturos, enquanto pneus mantidos no sol recebem a matéria orgânica diretamente com a chuva. A presença de maior diversidade de vegetação pode oferecer maiores oportunidades de sobrevivência para as larvas de culicídeos, mediante abrigo e alimentação, conforme verificado por FoRATTINI et al. (1998) em campos de cultivo de $\operatorname{arroz}$ (Tab. II).

Tabela II. Total de culicideos coletados $(\mathrm{N})$, número total de recipientes positivos para a presença de larvas de quarto instar e pupas $(T)$, densidade média de imaturos por criadouro e estação do ano (D) e média de recipientes positivos para larvas de quarto instar e pupas (X), nos três biótopos, na área rural de São José dos Pinhais, Paraná, Brasil, de novembro de 1997 a novembro de 1998.

\begin{tabular}{|c|c|c|c|c|c|c|c|c|c|c|c|c|}
\hline \multirow{2}{*}{ Estaçōes } & \multicolumn{4}{|c|}{ Interior de Mata } & \multicolumn{4}{|c|}{ Peridomicilio-sombra } & \multicolumn{4}{|c|}{ Peridomicilio-sol } \\
\hline & $N$ & $\mathrm{~T}$ & D & $X(D . P)$. & $\mathrm{N}$ & $T$ & D & $X(D . P)$. & N & $T$ & $D$ & $X(D . P)$. \\
\hline Verăo & 230 & 16 & 14,37 & $2,67(1,10) a$ & 1340 & 38 & 35,26 & $6,33(0,47) \mathrm{b}$ & 308 & 23 & 13,91 & $3,83(0,68) a$ \\
\hline Outono & 81 & 15 & 5,40 & $2,14(0,83) a^{\prime}$ & 900 & 29 & 31,03 & $4,14(1,81) a^{\prime}$ & 163 & 16 & 10,19 & $2,28(1,66) a^{\prime}$ \\
\hline Inverno & 333 & 19 & 17,52 & $2,71(0,70) a^{\prime \prime}$ & 500 & 31 & 16,13 & $4,47(0,90) \mathrm{b}^{\prime \prime}$ & 118 & 14 & 8,43 & $2,00(0,75) a^{\prime \prime}$ \\
\hline Primavera & 464 & 16 & 29,00 & $2,67(1,37) a^{\prime \prime \prime}$ & 796 & 16 & 49,75 & $2,67(1,37) a^{\prime \prime \prime}$ & 267 & 8 & 33,37 & $1,33(1,79) a^{\prime \prime \prime}$ \\
\hline Total & 1108 & 66 & 16,79 & $2,53(1,05)$ & 3536 & 114 & 31,01 & $4,38(1,78)$ & 856 & 61 & 14,03 & $2,35(1,59)$ \\
\hline
\end{tabular}

(a, a', a", a'") Letras diferentes na mesma estação indicam diferenças significativas (teste de Tukey, $p<0.05$ ), (D.P.): desvio padrão.

A análise do índice de dominância de Berger \& Parker, aplicados para os diferentes ecótopos agrupados estacionalmente, revelou maior predomínio de Culex eduardoi em dois ecótopos, interior de mata e peridomicílio com sombreamento, para os três tipos de recipientes testados, e apenas para o pneu introduzido no peridomicílio sem sombreamento. O mesmo índice aplicado ao trabalho realizado por Silva \& Lozover (1996) em área urbana, evidenciou maior predomínio de Culex quinquefasciatus Say, 1823 na ocupação dos recipientes nos dois ecótopos, durante praticamente todas as estações do ano. Durante o período do inverno, tanto no trabalho realizado por SiLVA \& Lozovel (1996) quanto em São José dos Pinhais, 
em área aberta apenas o pneu recebeu oviposições de mosquitos, no primeiro caso de Culex quinquefasciatus e no segundo de Culex eduardoi. (Tabs III, IV). A ocorrência de Culex eduardoi em pneu, durante o período do inverno, pode indicar maior capacidade desta espécie em resistir as baixas temperaturas, embora a correlação entre o número de imaturos desta espécie e a temperatura não tenha sido significativa, porém positiva. Os valores das correlações com as variáveis ambientais foram os seguintes: temperaturas médias $(0,3912)$, temperaturas máximas $(0,3179)$ e mínimas médias $(0,4277)$ e a precipitação pluviométrica acumulada $(0,2628)$. LOPES (1997) destaca a presença de Culex eduardoi em criadouros durante todo o ano e SiLVA \& Lozover (1996) indicam que a maior representatividade numérica de imaturos desta espécie foi coletada nos criadouros introduzidos no interior da mata, representados principalmente por pneus. Estes últimos autores ainda verificaram que o pneu sofre em menor intensidade a ação da temperatura, em contraposição ao plástico, fato que também poderia explicar a ocupação destes criadouros em área aberta durante o inverno.

Tabela III. Índice de Dominância de Berger \& Parker para os Culicidae coletados em recipientes antrópicos introduzidos na área rural de São José dos Pinhais, sob três condições, interior de mata, peridomicílio com sombreamento e sem sombreamento, de novembro de 1997 a novembro de 1998.

\begin{tabular}{|c|c|c|c|c|c|c|c|c|c|c|c|c|}
\hline \multirow{3}{*}{ Estações } & \multicolumn{4}{|c|}{ Interior da mata } & \multicolumn{4}{|c|}{ Peridomicilio com sombreamento } & \multicolumn{4}{|c|}{ Peridomicilio sem sombreamento } \\
\hline & \multicolumn{3}{|c|}{ Individualizados } & \multirow[t]{2}{*}{ Agrupados } & \multicolumn{3}{|c|}{ Individualizados } & \multirow[t]{2}{*}{ Agrupados } & \multicolumn{3}{|c|}{ Individualizados } & \multirow[t]{2}{*}{ Agrupados } \\
\hline & Pneu $F$ & lástic & Cerâmica & & Pneu & Plástico & Cerảmica & & Pneu & Plástico & Cerâmica & \\
\hline Verăo & $0,769^{*}$ & $1^{*}$ & $1,000^{* \star}$ & $0,730^{*}$ & $0,759^{\star}$ & $0,913^{\star}$ & $0,718^{*}$ & $0,793^{*}$ & $0,840^{*}$ & $1,000^{* * \star}$ & $0,760^{* \star *}$ & $0,386^{* * *}$ \\
\hline Outono & $0,931^{*}$ & $1^{*}$ & $1,000^{*}$ & $0,938^{*}$ & $0,671^{*}$ & $1,000^{*}$ & $1,000^{*}$ & $0,757^{*}$ & $0,852^{*}$ & $1,000^{* * *}$ & $1,000^{* * * *}$ & $0,429^{* * *}$ \\
\hline Inverno & $0,822^{*}$ & $1^{*}$ & - & $0,933^{*}$ & $0,903^{*}$ & $0,991^{*}$ & $0,964^{*}$ & $0,948^{*}$ & $0,991^{*}$ & - & - & $0,991^{*}$ \\
\hline Primavera & $0,900^{*}$ & $1^{*}$ & $0,566^{*}$ & $0,760^{*}$ & $0,637^{*}$ & $0,807^{*}$ & $0,938^{*}$ & $0,886^{*}$ & $0,652^{*}$ & $0,986^{* * *}$ & $1,000^{* * *}$ & $0,419^{* * *}$ \\
\hline
\end{tabular}

Espécies dominantes: $\left({ }^{\star}\right)$ Culex eduardoi, $\left({ }^{\star \star}\right)$ Culex acharistus, $\left({ }^{* \star *}\right)$ Culex grupo coronator.

Tabela IV. Aplicação dos Indices de Dominância de Berger \& Parker no trabalho de SILVA \& Lozover (1996) para os Culicidae coletados em área urbana de Curitiba, sob duas condições, interior de mata e em área adjacente à mata, no seguintes recipientes: pneu, plástico e cerâmica, de março de 1990 à março de 1991 .

\begin{tabular}{|c|c|c|c|c|c|c|c|c|}
\hline \multirow{3}{*}{ Estações } & \multicolumn{4}{|c|}{ Interior da mata } & \multicolumn{4}{|c|}{ Área adjacente à mata } \\
\hline & \multicolumn{3}{|c|}{ Individualizados } & \multirow[t]{2}{*}{ Agrupados } & \multicolumn{3}{|c|}{ Individualizados } & \multirow[t]{2}{*}{ Agrupados } \\
\hline & Pneu & Plástico & Cerâmica & & Pneu & Plástico & Cerâmica & \\
\hline Verăo & $0,874^{*}$ & - & $0,651^{*}$ & $0,776^{*}$ & $1,000^{*}$ & $1^{*}$ & $1,000^{*}$ & $1,000^{*}$ \\
\hline Outono & $1,000^{*}$ & - & $1,000^{*}$ & $1,000^{*}$ & $1,000^{\star}$ & $1^{*}$ & $0,836^{\star}$ & $0,963^{*}$ \\
\hline Inverno & - & - & - & - & $0,998^{*}$ & - & - & $0,998^{\star}$ \\
\hline Primavera & $0,976^{*}$ & - & $1,000^{*}$ & $0,976^{*}$ & $1,000^{*}$ & - & - & $1,000^{*}$ \\
\hline
\end{tabular}

Espécie dominante: $\left({ }^{*}\right)$ Culex quinquefasciatus, (1) indice aplicado para cada tipo de recipiente, (2) índice aplicado ao somatório dos dados obtidos nos criadouros.

A análise do índice de dominância para cada um dos tipos de recipientes revela que a ocupação de criadouros por Culex grupo coronator restringiu-se quase que exclusivamente ao peridomicílio sem sombreamento. Esta espécie ocupou

Revta bras. Zool. 18 (Supl. 1): 51 - 60, 2001 
preferencialmente os recipientes constituídos por plástico e cerâmica, estando presente durante a primavera, verão e outono. A ocupação de recipientes instalados em locais onde a queda de matéria orgânica vegetal é reduzida, pode demonstrar maior flexibilidade de Culex grupo coronator em explorar ambientes antrópicos. Em investigação, realizada por SiLvA \& LOZOVEI (1996), os números mais expressivos de imaturos desta espécie, foram coletados em criadouros instalados em área sem sombreamento. (Fig. 2, Tab. III).

Culex acharistus, ocorreu com maior freqüência no período do verão, explorando os pneus nos três tipos de condições em que foram instalados, e apresentando reduzida preferência em explorar recipientes instalados sob insolação direta, com exceção dos pneus (Tab. III, Fig. 2). As espécies Culex acharistus e Culex grupo coronator embora apresentem preferências em explorar criadouros naturais de mesma natureza, representados por coleções líquidas no solo (FORATTINI 1965), não exploraram da mesma forma os criadouros artificiais estudados, com a ocorrência simultânea sendo evento de baixa freqüência.

O número de espécies de Culicidae coletadas em recipientes artificiais introduzidos em capão de mata na área urbana por SILVA \& LozovEI (1996) foi inferior ao registrado durante as coletas na área rural de São José dos Pinhais. Havendo em comum quatro espécies, com exceção de Culex quinquefasciatus não coletada no presente estudo, assim como Culex bigoti e Limatus durhamii coletadas apenas na área rural. LOURENÇO-DE-OLIVEIRA \& HEYDEN (1986) destacam que Culex quinquefasciatus é predominante em cidades, vilas e demais aglomerados humanos, tornando-se rara em locais com baixa densidade humana, por exemplo em zona rural com habitações esparsas. LOPES et al. (1993) destacam que Limatus durhamii prefere explorar recipientes localizados em áreas sombreadas, costumando ser a espécie mais freqüente em chácaras e ambientes periurbanos, o que pode indicar características de hábito silvestre. Culex bigoti na presente investigação, ocorreu apenas em área rural, sendo encontrada colonizando recipientes do tipo pneu e plástico, este último pela primeira vez apontado como criadouro alternativo para esta espécie. As demais espécies também foram encontradas em mata na área urbana, através de diferentes procedimentos de coleta, o que revela em alguns casos comportamento ambíguo. Aedes terrens, no presente estudo, colonizou pneus, no entanto, SILVA \& LOzOVEI (1996), embora tenham estabelecido condições similares ao desenvolvido em área rural, coletaram-na apenas em oco de árvore, criadouro considerado como natural para esta espécie por DAVIS (1944) e GOMES et al. (1992). BAUMGARTNER (1988) destaca que espécies que exploram criadouros naturais em determinados locais, podem ocupar recipientes artificiais, como por exemplo pneus, com sucesso. Esta mudança pode ser potencializada pela semelhança da comunidade de culicídeos em pneus e ocos de árvore, como apontado por BEIER et al. (1983) para a América do Norte. A presença do culícideo do gênero Phoniomyia Theobald, 1903, também foi detectada através de métodos diferentes, em criadouro artificial na área rural e isca humana em mata na área urbana (SILVA \& LOZOVEI 1998).

A natureza dos materiais descartados pela atividade antrópica, seja em área rural ou urbana, apresenta as mesmas características, no entanto, a colonização por espécies de Culicidae pode apresentar dinâmica diferenciada, devido às exigências 
58

Calado \& Silva
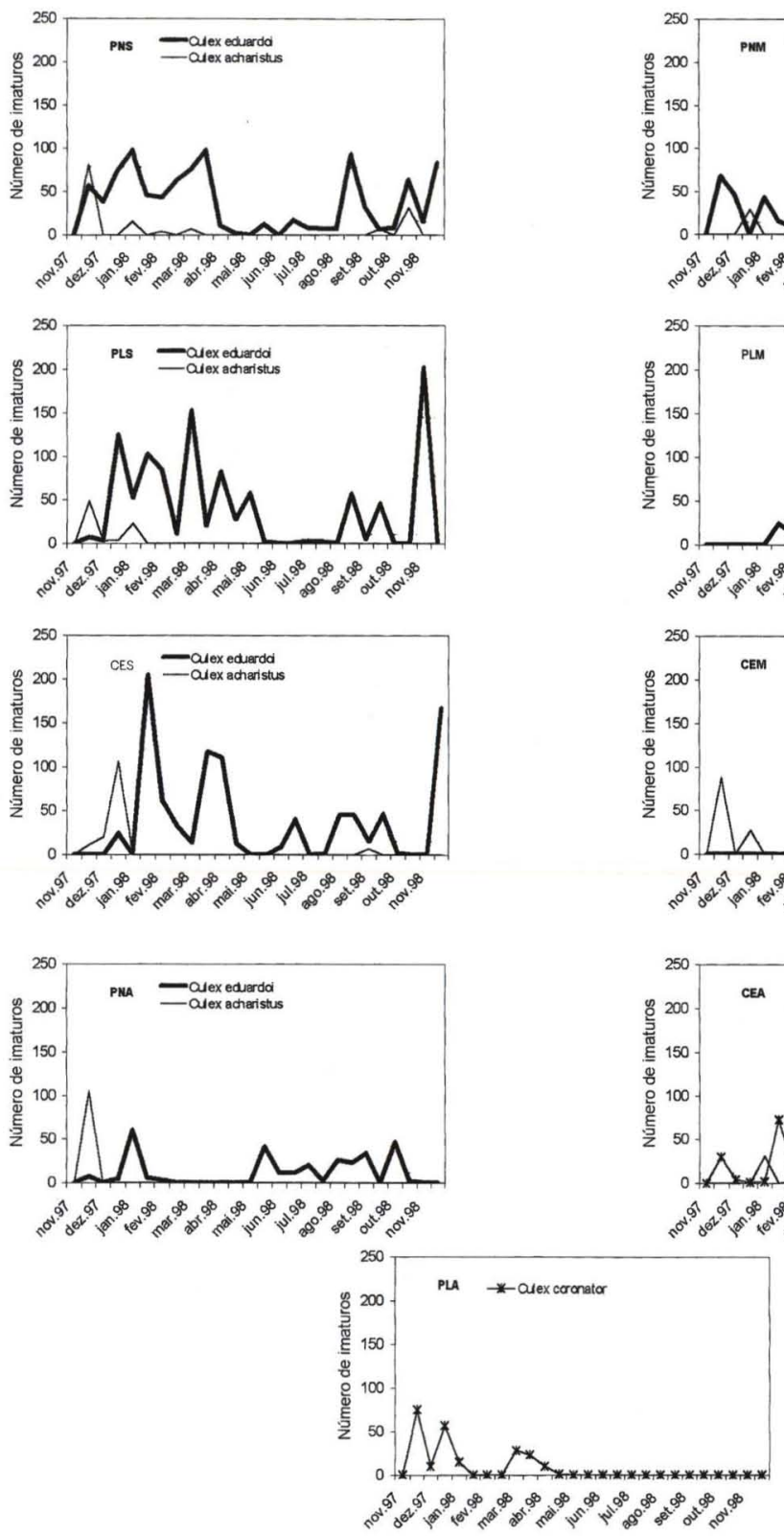
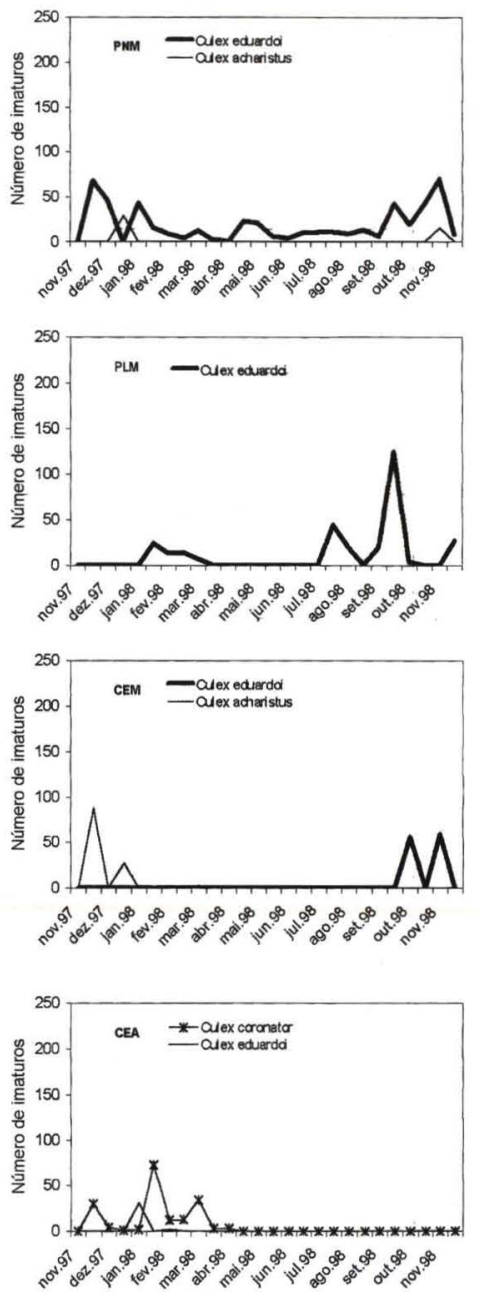

Fig. 2. Flutuação das espécies de Culicidae mais abundantes nos três tipos de recipientes artificiais e nos três ecótopos de instalação, em área rural de São José dos Pinhais. (PNS) Pneu com sombreamento, (PNM) pneu em interior de mata, (PNA) pneu sem sombreamento, (PLS) plástico com sombreamento, (PLM) plástico em interior de mata, (PLA) plástico sem sombreamento, (CES) cerâmica com sombreamento, (CEM) cerâmica em interior de mata, (CEA) cerâmica sem sombreamento.

Revta bras. Zool. 18 (Supl. 1): 51 - 60, 2001 
ambientais específicas que determinam o sucesso das diferentes espécies de culicídeos. Diferenças observadas na colonização dos criadouros de mesma natureza, mas instalados em áreas distintas, podem ser determinadas pela paisagem florística local, representada pela matéria orgânica disponível à alimentação e proteção dos imaturos. DORVILLÉ (1996) destaca que embora grande parte dos estudos realizados estejam focados no comportamento de domiciliação das espécies de culicídeos, pouca informação tem sido gerada em relação às influências provocadas pela atividade humana. A introdução de recipientes antrópicos pode não evidenciar a riqueza de espécies de culicídeos presentes em determinada área, mas pode indicar as espécies que apresentam maior facilidade de adaptação às modificações ambientais produzidas pela atividade humana e como estas alterações influenciam a ocupação dos criadouros.

AGRADECIMENTO. Ao SIMEPAR (Sistema Meteorológico do Paraná) pelo fornecimento dos dados meteorológicos utilizados na elaboração deste trabalho.

\section{REFERÊNCIAS BIBLIOGRÁFICAS}

Comec. 1984. Dados básicos da Região Metropolitana de Curitiba. Curitiba, Prefeitura Municipal de Curitiba, Vol. 1.

BAUMgaRTNER, D.L. 1988. Suburban accumulations of discarded tires in northern Illinois and their associated mosquitoes. J. Am. Mosq. Control Assoc. 4 (4): 500-508.

Beier, J.C.; N. Travispatricoski C.E. \& J. Kranzfelder. 1983. Habitat segregation among larval mosquitoes (Diptera: Culicidae) in tire yards in Indiana. Jour. Med. Entol. 20 (1): 76-80.

DAvis, D.E. 1944. Larval habitats of some brazilian mosquitoes. Rev. Entomol. 15 (1-2): 221-235.

DORVILLÉ, L.F.M. 1996. Mosquitoes as bioindicators of forest degradation in southeastern Brazil, a statistical evaluation of published data in literature. Stud. Neotrop. Fauna Environm. 31: 86-78.

Forattini, O.P. 1965. Entomologia Médica. São Paulo, Faculdade de Higiene e Saúde Pública, Vol. 2., 506p.

1998. Mosquitos Culicidae como vetores emergentes de infecções. Rev. Saúde Públ. 32 (6): 497-502.

Forattini, O.P.; I. KAKITANi; G.R.A.A.M. Marques \& M. DE Brito. 1998. Formas imaturas de anofelineos em recipientes artificiais. Rev. Saúde Públ. 32 (2): 189-191.

Gomes, A. DE C.; O.P. Forattini; I. Kakitani; G.R.A.M. Marques; C.C. De A. Marques.; D. Marucci; \& M. DE BRITO. 1992. Microhabitats de Aedes albopictus (Skuse) na região do Vale do Paraíba, Estado de São Paulo, Brasil. Rev. Saúde Públ. 26 (2): 108-118.

Harbach, R.E. \& I.J. Kitciing. 1998. Phylogeny and classification of the Culicidae (Diptera). Syst. Entomol. 23: 372-370.

LOPES, J. 1997. Ecologia de mosquitos (Diptera: Culicidae) em criadouros naturais e artificiais de área rural do Norte do Estado do Paraná, Brasil. V. Coletas de larvas em recipientes artificiais instalados em mata ciliar. Rev. Saúde Públ. 31 (4): 370-377.

Lopes, J.; M.A.N. DA Silva; A.M Borsato; V.D.R.B. de Oliveira \& F.J. DE A. Oliveira. 1993. Aedes (Stegomyia) aegypti e a culicideofauna associada em área urbana da região sul, Brasil. Rev. Saúde Públ. 27 (5): 326-333.

LOURENÇO-DE-Oliveira, R. \& R. HEydEn. 1986. Alguns aspectos da ecologia dos mosquitos (Diptera: Culicidae) de uma área de planície (Granjas Calábria), em Jacarepaguá, Rio de Janeiro. IV. Preferências alimentares quanto ao hospedeiro e frequiência domiciliar. Mem. Inst. Oswaldo Cruz $81(1): 15: 27$.

Luz, E.; J. Consol.im; O.C. Barbosa \& P.B. Torres. 1987. Larvas de Anopheles (Subgênero Kerleszia) 
Theobald 1905 encontradas em criadouros artificiais, no estado do Paraná, Brasil. Rev. Sáude Públ. 21 (5): 466-468.

MagurRan, A.E. 1988. Ecological Diversity and its Measurement. Princeton, Princeton Univ. Press, $179 \mathrm{p}$.

Silva, A.M. DA \& R.M.T. DA MEnezes. 1996. Encontro de Aedes scapularis (Diptera: Culicidae) em criadouro artificial em localidade da região Sul do Brasil. Rev. Saúde Públ. 30 (1): 103-104.

Silva, M.A.N. DA \& A.L. Lozovel. 1996. Criadouros de imaturos de mosquitos (Diptera, Culicidae) introduzidos em mata preservada na área urbana de Curitiba, Paraná, Brasil. Revta bras. Zool. 13 (4): $1023-1042$.

1998. Mosquitos (Diptera, Culicidae) capturados com isca humana em área preservada de Curitiba, Paraná. Revta bras. Zool. 15 (4): 965-976.

Recebido em 10.VIII.2000; aceito em 15.VI.2001. 